\title{
Sindrome delle apnee ostruttive del sonno nell'acromegalia: revisione sistematica e meta-analisi della letteratura
}

\author{
Giovanna Mantovani $^{1} \cdot$ Alessandra Mangone $^{1}$
}

Accettato: 27 gennaio 2021 / Pubblicato online: 6 agosto 2021

(c) The Author(s) 2021

\section{Commento a:}

Obstructive sleep apnea in acromegaly and the effect of treatment: a systematic review and meta-analysis. M. Parolin, F. Dassie, L. Alessio, A. Wennberg, M. Rossato, R. Vettor, P. Maffei, C. Pagano. J Clin Endocrinol Metab 105(3):e23-e31

I pazienti affetti da acromegalia presentano un aumento di mortalità e morbilità rispetto alla popolazione generale, che è principalmente riconducibile alle complicanze cardiovascolari, respiratorie e neoplastiche che caratterizzano la patologia. Le alterazioni respiratorie si manifestano frequentemente nella forma di sindrome delle apnee ostruttive del sonno (Obstructive Sleep Apnea Syndrome, OSAS), definita come un'alterazione della ventilazione notturna con conseguente eccessiva sonnolenza diurna, che si riscontra in una percentuale compresa tra il 20 e 1'80\% dei pazienti acromegalici [1]. Tale sindrome può presentarsi in forma ostruttiva, la più frequente, dovuta a un collasso delle vie aeree superiori che è legato, nei pazienti acromegalici, all'edema dei tessuti molli e alle modificazioni oro-facciali tipiche quali macroglossia, prognatismo e ipertrofia laringea o, più raramente, in forma centrale, data da un'alterazione dei centri di controllo della respirazione ad opera dell'eccesso di GH mediante aumentato tono somatostatinergico [1]. L'OSAS si definisce col riscontro di un indice di apneaipopnea (Apnea-Hypopnea Index, AHI) > 5 eventi/ora, misurato tramite polisonnografia, che ne rappresenta il gold standard diagnostico. Le linee guida raccomandano di sottoporre i pazienti acromegalici, sia alla diagnosi sia durante

G. Mantovani

giovanna.mantovani@unimi.it

1 Unità di Endocrinologia, Fondazione IRCCS Ca' Granda Ospedale Maggiore Policlinico; Dipartimento di Scienze Cliniche e di Comunità, Università degli Studi di Milano, Milano, Italia il follow-up di malattia, a screening per OSAS mediante scala di Epworth per la valutazione della sonnolenza (Epworth Sleepiness Scale, ESS) e, quindi, a polisonnografia [2]. La sindrome respiratoria non sempre si risolve con la remissione della patologia acromegalica: i molteplici studi che hanno analizzato la stretta relazione tra acromegalia e OSAS hanno mostrato spesso risultati discordanti in merito.

La presente meta-analisi ha indagato il rapporto tra apnee ostruttive e acromegalia in letteratura, studiando in particolare il ruolo dell' attività di malattia e l'effetto dei trattamenti sul rischio di OSAS dei pazienti. Sono stati selezionati 24 studi e scelti come outcome di interesse la prevalenza di OSAS e l'AHI nei pazienti acromegalici in fase attiva $v s$ inattiva di malattia in studi trasversali, e l'AHI pre e post trattamento, sia chirurgico che medico, in studi longitudinali a breve termine (mediana 6 mesi).

Per quanto riguarda il primo outcome, non si sono registrate differenze significative nella prevalenza di OSAS nei pazienti acromegalici con malattia attiva rispetto a inattiva, né nell'AHI in tali categorie di pazienti. Gli autori hanno attribuito questo risultato a un'incompleta reversibilità del danno ormonale sulle vie respiratorie superiori suggerendo, inoltre, un contributo nella patogenesi dell'OSAS delle altre comorbidità dell'acromegalia e/o delle complicanze conseguenti alla terapia come, ad esempio, l'ipopituitarismo post-chirurgico.

In merito, invece, all'analisi dell'AHI prima e dopo il trattamento, in apparente contrasto con quanto evidenziato dall'analisi degli studi trasversali, la meta-analisi ha rivelato un significativo miglioramento nei pazienti trattati in termini di severità dello stesso $(p<0,0001)$, indipendentemente dal tipo di approccio (medico o chirurgico) utilizzato. A proposito di questo riscontro, gli autori hanno ipotizzato un transitorio effetto benefico del trattamento sull'edema tissutale, che si perde nel lungo termine. Inoltre, questo 
risultato può essere ricondotto a una diminuzione effettiva dell'AHI che, tuttavia, si mantiene comunque a livelli diagnostici per sindrome OSAS anche dopo la terapia. Vanno poi tenuti in considerazione il breve periodo di trattamento analizzato e l'assenza di un gruppo di controllo negli studi longitudinali. Bisogna menzionare, infine, che lo scarso utilizzo della scala clinica ESS al follow-up nella maggior parte degli studi clinici inclusi ha comportato la necessità di analizzare unicamente l'AHI come indice di miglioramento, tenendo quindi poco in considerazione l'aspetto soggettivo sull'impatto delle apnee del sonno nella vita quotidiana.

In conclusione, la prevalenza di OSAS è risultata simile nei pazienti con malattia acromegalica in fase attiva e inattiva; tuttavia, gli studi longitudinali hanno suggerito che il trattamento potrebbe migliorarne l'entità. Questi risultati ribadiscono l'importanza della diagnosi precoce di acromegalia, nonché la necessità di seguirne con attenzione le comorbidità, riservando una particolare attenzione al rischio di OSAS anche nei pazienti con malattia ben controllata, in considerazione dell'impatto che questa sindrome respiratoria presenta sul rischio cardiovascolare e sulla qualità della vita dei pazienti. Sarebbero utili studi futuri con disegni di tipo randomizzato per comparare l'efficacia dei trattamenti nei pazienti acromegalici anche in termini di miglioramento delle complicanze respiratorie.
Funding Note Open access funding provided by Università degli Studi di Milano within the CRUI-CARE Agreement.

Nota della casa editrice Springer Nature rimane neutrale in riguardo alle rivendicazioni giurisdizionali nelle mappe pubblicate e nelle affiliazioni istituzionali.

Open Access This article is licensed under a Creative Commons Attribution 4.0 International License, which permits use, sharing, adaptation, distribution and reproduction in any medium or format, as long as you give appropriate credit to the original author(s) and the source, provide a link to the Creative Commons licence, and indicate if changes were made. The images or other third party material in this article are included in the article's Creative Commons licence, unless indicated otherwise in a credit line to the material. If material is not included in the article's Creative Commons licence and your intended use is not permitted by statutory regulation or exceeds the permitted use, you will need to obtain permission directly from the copyright holder. To view a copy of this licence, visit http://creativecommons.org/licenses/by/4.0/.

\section{Bibliografia}

1. Parolin M, Dassie F, Alessio L et al (2020) Obstructive sleep apnea in acromegaly and the effect of treatment: a systematic review and meta-analysis. J Clin Endocrinol Metab 105(3):e23-e31

2. Melmed S, Casanueva FF, Klibanski A et al (2013) A consensus on the diagnosis and treatment of acromegaly complications. Pituitary 16(3):294-302 